\title{
IRREGULAR BALL-QUOTIENT SURFACES WITH NON-POSITIVE KODAIRA DIMENSION
}

\author{
AleKsander Momot \\ Dedicated to Rolf-Peter Holzapfel on occasion of his retirement
}

\begin{abstract}
Let $\Gamma \subset \mathbf{P U}(2,1)$ be a lattice. $\Gamma$ then acts on the complex open unit ball $\mathbf{B} \subset \mathbb{C}^{2}$. Suppose that $\Gamma$ is not cocompact and 'sufficiently' neat. Then there exists a compactification $X=\overline{\Gamma \backslash \mathbf{B}}$ with the property that $X$ is a smooth projective surface and $D=X \backslash(\Gamma \backslash \mathbf{B})$ is a disjoint sum of elliptic curves. The main result is: $\operatorname{kod}(X) \leq 0$, $h^{1}\left(\mathcal{O}_{X}\right)>0 \Leftrightarrow X$ admits an abelian surface $Y$ as minimal model, and $Y$ carries a divisor $S=\sum S_{i}$ which consists of elliptic curves such that $4 \cdot|\operatorname{sing} S|=\sum\left|S_{i} \cap \operatorname{sing} S\right|$.
\end{abstract}

\section{Introduction}

1.1. Motivation. Let $U$ be a complex manifold which has the complex open unitball $\mathbf{B} \subset \mathbb{C}^{2}$ as universal holomorphic covering. If $U$ is compact then it is of general type. The situation changes if one considers surfaces $X$ which are toroidal compactifications of non-compact unramified ball-quotients $U \subset X$. Then the compactification divisor $D=X \backslash U$ is a disjoint sum of elliptic curves and $X$ may be of special type. The article is concerned with this problem. It marks the beginning of a classification of surfaces $X=U \cup D$ by purely geometric methods. For simplicity, the class of such surfaces will be denoted by $\mathcal{T}$.

Recall that $\mathbf{P} \mathbf{U}(2,1)$ is identified with the group of holomorphic automorphisms of

$$
\mathbf{B}=\left\{\left[x_{0}: x_{1}: x_{2}\right] \in \mathbf{P}^{2} ;\left|x_{0}\right|^{2}+\left|x_{1}\right|^{2}<\left|x_{2}\right|^{2}\right\}
$$

in a natural way. Therefore, surfaces in $\mathcal{T}$ typically arise from the minimal singularity resolution of Baily-Borel compactifications of ball-quotients $U=\Gamma \backslash \mathbf{B}$ by neat subgroups $\Gamma \subset \mathbf{P U}(2,1)$ which are arithmetic but not cocompact. Natural candidates are subgroups $\Gamma \subset \mathbf{P} \mathbf{U}\left(2,1 ; \mathcal{O}_{\mathbf{K}}\right)$ with $\mathbf{K}$ an imaginary quadratic field, and their quotients serve as a natural pendant to Hilbert modular surfaces. More generally, however, any discrete $\Gamma \subset \mathbf{P U}(2,1)$ contains a neat subgroup of finite index inducing a surface in $\mathcal{T}$, provided that $\Gamma \backslash \mathbf{B}$ is not compact and admits a fundamental domain with finite Bergman-volume. Hence, $\mathcal{T}$ forms in fact the class of 'generic' compactified ball-quotients.

The french mathematician E. Picard discovered the ball 125 years ago as co-domain

Received by the editors November 25, 2007.

2000 Mathematics Subject Classification. Primary 11J25; Secondary 14G35.

Key words and phrases. special surfaces, modular and Shimura varieties, Picard modular surfaces. 
of multivalued hypergeometric integrals and also noticed the analogy to the $j$-line in the theory of elliptic functions (cf. [9]). Systematic research in this area was, among others, started by Holzapfel. He called the surfaces, which arise from subgroups $\Gamma \subset \mathbf{P U}\left(2,1 ; \mathcal{O}_{\mathbf{K}}\right)$, Picard modular surfaces. Already in the middle of the 1980's, Holzapfel was able to boast an amount of classification results for these special ball-quotients. We refer to [4] and to the new book [8] for an overview and a bibliography. These results, however, are based on an arithmetic theory of ball-lattices and are proved using arithmetically defined lattice invariants. By way of contrast, the fundamental work of Deligne-Mostow [2] shows that also non-arithmetic ball-lattices should be considered. Moreover, even for the arithmetic case it is not clear to what extent there are general obstructions for ball-quotient surfaces with respect to the Enriques-Kodaira classification.

1.2. Statement and discussion of the main result. In the hypotheses of our main theorem all complex surfaces are smooth and compact.

Theorem 1.1. Let $Y$ be a relatively minimal surface with non-positive Kodaira dimension. Assume that $Y$ is irregular, that is, $h^{1}\left(\mathcal{O}_{Y}\right)>0$. Then the following are equivalent:

(1) $Y$ is birationally dominated by a surface in $\mathcal{T}$.

(2) $Y$ is an abelian surface. It supports a reduced elliptic divisor $S=\sum S_{i}$ satisfying the proportionality condition

$$
4|M|=\sum s_{i}
$$

where $M=\operatorname{sing} S$ is the set of singular points of the curve $S$ and $s_{i}=\left|S_{i} \cap M\right|$.

If (2) holds then $Y$ is isogenous to a product $C \times C$ of an elliptic curve $C$, and we have: $s_{i}>0$ for each $i ; s_{x}=\left|\left\{S_{i} ; x \in S_{i}\right\}\right| \geq 3$ for $x \in M$; blowing up once along each $x \in M$ produces a surface in $\mathcal{T}$ with compactification divisor the proper transform of $S$.

The proof relies on two steps. We will show that an $X \in \mathcal{T}$ with Kodaira dimension $\operatorname{kod}(X) \leq 0$ admits only abelian minimal models or is regular. This assertion is completely new. Then, up to the claim about $s_{i}, s_{x}$ (which results from Cor. 3.2 and Lemma 3.3), the gap from the latter result to the stated theorem is closed by the following criterion of Holzapfel [7, Thm. 2.5]:

Theorem 1.2 (Holzapfel). Let $Y$ be an abelian surface admitting a reduced divisor $S=\sum S_{i}$ of elliptic curves. Let $M=\operatorname{sing} S$ be the set of singular point of $S$ and set $s_{i}=\left|S_{i} \cap M\right|$. Then $Y$ is the minimal model of an $X \in \mathcal{T}$ with compactification divisor $D$ the proper transform of $S$ if and only if the porportionality condition

$$
4|M|=\sum s_{i}
$$

holds with $s_{i}>0$ for each $i$. More precisely, if the conditions are satisfied then $X$ results from blowing up once along each point $x \in M$ and $Y$ is isogenous to a product $C \times C$ of an elliptic curve $C$.

The value of Thm. 1.1 and Thm. 1.2 arises from the following modular intepretation. For $m \geq 2$, the vector space $[\Gamma, m]$ of $\Gamma$-modular forms of weight $m \geq 2$ is naturally identified with $H^{0}\left(X, m\left(K_{X}+D\right)\right)$. Now, together with the surfaces $X$ and $Y$, to 
a large extend the corresponding rings of automorphic forms can also be effectively compared to each other. The automorphic forms belonging to a square $C \times C$ of an elliptic curve $C$ may be expressed by means of the classical theta function, and hence the $\Gamma$-automorphic forms coming from the ball-lattice $\Gamma$ corresponding to $X$ may be 'transferred' into such 'theta-expressions'. In [6], Holzapfel laid the foundation for this approach by constructing 'appropriate' quotients of elliptic theta functions and illustrated the advantages of such representations for the determination of $X$ and the ring of $\Gamma$-automorphic forms. In [5], he also calculated the dimension of $[\Gamma, m]$ in the abelian case. The proof given there readily extends to the general case. To be more precise, we have

$$
\operatorname{dim}_{\mathbb{C}}[\Gamma, m]=\frac{3}{2} m(m-1) e(X)+\chi(X)+h_{X} \quad(m \geq 2)
$$

where $e(X)$ is the topological Euler number, $\chi(X)=\sum(-1)^{i} h^{i}\left(X, \mathcal{O}_{X}\right)$ and $h_{X}$ is the number of components of the compactification divisor $D$.

However, it is known that 'most' surfaces in $\mathcal{T}$ are of general type. The log pair $(X, D)$ is logarithmically of general type, i.e. $\operatorname{kod}\left(X, K_{X}+D\right)=2$. Thus our structure result seems to deal with exceptional cases. And in fact, up to isogeny only few commensurability classes of surfaces in $\mathcal{T}$ of special type are known yet; all of them admit abelian minimal models (cf. [7]).

1.3. Organization of the proof. The proof of Thm. 1.1 is essentially based on classification theory of algebraic surfaces. In order to make our result more accessible for researchers who, for instance, come from arithmetic but are no experts in geometric methods, we repeat in Section 2 some general facts from the geometry of surfaces. In Section 3 we state first properties of surfaces in $X \in \mathcal{T}$ which are needed in all parts of the proof. In the two remaining sections we prove our main result. After the preparations, each step is rather easy.

\section{Short review of surface theory}

2.1. From the classification theory of algebraic surfaces. We focus on the category of algebraic surfaces over $\mathbb{C}$ which are non-singular, integral and projective. Throughtout, a 'surface' will always mean a two dimensional $\mathbb{C}$-variety of this type as long as the converse is not explicitly stated. A surface $X$ is called relatively minimal if it does not contain a $(-1)$-curve $L$, i.e. a rational curve with self-intersection $=-1$. It is a minimal surface if all relatively minimal models in the birational class of $X$ are pairwise isomorphic. The latter holds automatically if the Kodaira dimension $\operatorname{kod}(X)$ is non-negative. Starting with an arbitrary smooth surface $X$, one obtains a birational morphism to a relatively minimal model by succesively blowing down arbitrary (-1)- curves. For this and what follows, we refer to [1]. A geometrically ruled surface is by definition a smooth surface $X$ together with a $\mathbf{P}^{1}$-bundle $\pi: X \longrightarrow C$ over an irreducible smooth curve $C$. More generally, a fibration that factors through a geometrically ruled surface is called a ruling, and a ruled surface is a surface together with a ruling. By classification theory for algebraic surfaces we have: 
(1) If $\operatorname{kod}(X)=-\infty$ and $q(X)>0$ then $X$ admits a ruling over a curve $C$ with the property that $q(X)=g_{C}$, the genus of $C$. In this case, $X$ is birational to $\mathbf{P}^{1} \times C$. Moreover, $N$ um $(X)$, the group of divisors modulo numerical equivalence, is free of rank 2 and generated by a fibre $F$ and an arbitrary section $C_{o}$ of $\pi$ (cf. [3, Ch. V, Prop. 2.3]).

(2) If $X$ is a minimal surface so that $\operatorname{kod}(X)=0$ then $K_{X}$ is numerically trivial. If $q(X)>0$ then a minimal surface $X$ is either abelian, or a hyperelliptic surface. In the second case, there exists an etale covering $A \longrightarrow X$ by an abelian surface $A$, and $b_{2}(X)=2$.

2.2. A result on ruled surfaces. We will need the following easy lemma.

Lemma 2.1. Let $\pi: X \longrightarrow C$ be a ruled surface. Let $C_{o} \subset X$ be a section of $\pi$. Then there exists a diagram $X \stackrel{\phi}{\longrightarrow} Y \stackrel{\tilde{\pi}}{\longrightarrow} C$ such that $\phi$ is an isomorphism in an open neighbourhood of $C_{o}$ and $\tilde{\pi}: Y \longrightarrow C$ is a geometrically ruled surface.

Proof. Let $F=\sum E_{k}$ be a reducible $\pi$-fibre. Since $F C_{o}=1$, there is precisely one $E_{k_{o}}$ having non-trivial intersection with $C_{o}$. Therefore, we have to show that one can find a succession of blowing downs of the $E_{k}$ 's with the property that the image of $E_{k_{o}}$ becomes a smooth fibre. By induction on the number $n$ of components $E_{k}$, all we have to verify is the following: As long as $n \geq 2, F=\sum E_{k}$ supports one (-1)-curve $E_{k_{1}}$ with $E_{k_{1}} C_{o}=0$. The assertion is certainly clear if $n=2$. Assume the assertion holds for a $n \geq 2$, and let $F_{n}$ be a fibre consisting of $n$ components. Blowing up once along $x \in F_{n}$, the resulting (-1)-curve satifies our requirement if $x \notin C_{o}$. If $x \in C_{o}$ let $E_{k_{1}} \neq E_{k_{o}}$ be a (-1)-curve not intersecting $C_{o}$. Then the proper transform of $E_{k_{1}}$ on the new fibre $F_{n+1}$ is still a (-1)-curve not intersecting (the proper transform of) $C_{o}$. The assertion follows, because each fibre with $n+1$ components is a blown up $F_{n}$.

\section{Fundamental properties of surfaces in $\mathcal{T}$}

We return to toroidally compactified ball-quotient surfaces. The most important properties of surfaces in $\mathcal{T}$ are summarized in the following theorem. It is a special case of Tian-Yau's inequality [10, Thm. 3.1].

Theorem 3.1. Let $X$ be a complex projective surface together with a normal-crossing divisor $D \subset X$. Assume:

(1) $K_{X}+D$ is nef and $K_{X}+D$ is ample modulo $D$.

(2) $3 c_{2}(X)=\left(K_{X}+D\right)^{2}>0$.

Then $X \backslash D$ admits the open unit ball $\mathbf{B} \subset \mathbb{C}^{2}$ as universal holomorphic covering. Conversely, an unramified ball-quotient surface $X \backslash D$ with compactification divisor $D$ which is a sum of elliptic curves fulfills (1) and (2).

In (1), 'ample modulo $D$ ' means that $\mathcal{O}_{C}\left(K_{X}+D\right)$ is positive for each curve $C \subset X$ not supported in $D$. (1) is equivalent to the fact that for large $m$ the sheaf $\mathcal{L}^{\otimes m}$, where $\mathcal{L}=\mathcal{O}_{X}\left(K_{X}+D\right)$, is generated by global sections and provides an embedding of $X \backslash D$ into projective space (cf. [10, Rem. p. 613]). 
Corollary 3.2. Let $X$ be a surface in $\mathcal{T}$ with compactification divisor $D$. Then $D$ is contractible such that the contraction space is a normal projective variety. In particular, each component $D_{i}$ of $D$ has a negative self-intersection number $D_{i}^{2}<0$.

Although readily verified, the next two observations are indispensible ingredients in the proof of our main result.

Lemma 3.3. Let $X$ be in $\mathcal{T}$ with compactification divisor $D$ and consider a rational curve $L \subset X$. Then $|L \cap D| \geq 3$.

Proof. Assume $|L \cap D|=2$. Then $L \backslash D$ is up to isomorphism $\mathbb{C}^{*}$, and admits the complex line a universal holomorphic covering. Similarly, if $|L \cap D|=1$ then $L \backslash D \simeq \mathbb{C}$. Proceeding to universal coverings we would thus obtain a holomorphic map from $\mathbb{C}$ to $\mathbf{B}$, which is not trivial. This contradicts Liouville's theorem.

Lemma 3.4. Let $\pi: X \longrightarrow C$ be a fibration of an element in $\mathcal{T}$ over an elliptic curve $C$. Assume that $\phi(D)=C$. Then there exists a commutative diagram

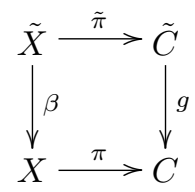

such that $\beta$ and $g$ are etale; that is, $\tilde{X}$ is a surface in $\mathcal{T}$ with compactification divisor $\tilde{D}=\beta^{-1}(D)$ and fibred over $\tilde{C}$, and with the additional property that each component of $\tilde{D}$ is a section of $\tilde{\pi}$, as soon as it dominates $\tilde{C}$.

Proof. Let $F$ be a general fibre of $\pi$. The proof is by induction on $n(X):=$ $\max \left\{D_{i} F\right\}$, where $D_{i}$ ranges over all curves in the compactification divisor dominating $C$. We suppose w.l.o.g. that $n(X)=D_{1} F>1$, since otherwise there is nothing to prove. By general properties of the fibre product, the projection

$$
\pi_{1}:=\operatorname{pr}_{D_{1}}: X_{1}=X \times_{C} D_{1} \longrightarrow C_{1}=D_{1}
$$

has a copy $T_{1} \subset X_{1}$ of $D_{1}$ as a section. One checks that the projection $\beta_{1}: X_{1} \longrightarrow X$ is etale, and that a general fibre of $\pi$ is dominated by $n(X)=\operatorname{deg}\left(D_{1} \rightarrow C\right)$ fibres of $\pi_{1}$. Therefore, $X_{1}$ is smooth, $\operatorname{deg} \beta_{1}=n(X)$, and $\beta_{1}^{-1}\left(D_{1}\right)$ is of the form $T_{1}+B$ with a divisor $B$. More precisely, noting that $\beta_{1}^{-1}(X \backslash D)$ must have the ball $\mathbf{B}$ as universal holomorphic covering, one recognizes that $X_{1}$ is a surface in $\mathcal{T}$ with compactification part $\beta_{1}^{-1}(D)$. Hence, $B$ consists of a disjoint sum of elliptic curves contained in the compactification divisor of $X_{1}$. Fix a $D_{i}$ dominating $C$ and let $D_{i j}$ range over the curves in $\beta_{1}^{-1}\left(D_{i}\right)$. We claim:

$$
\sum_{j} \operatorname{deg}\left(D_{i j} \rightarrow C_{1}\right)=\operatorname{deg}\left(D_{i} \rightarrow C\right)(i \text { fixed })
$$

We have $\operatorname{deg}\left(D_{1} \rightarrow C\right)=\operatorname{deg}\left(C_{1} \rightarrow C\right)$. Thus, an easy calculation yields for all $j$ :

$$
\operatorname{deg}\left(D_{i j} \rightarrow D_{i}\right) \cdot \operatorname{deg}\left(D_{i} \rightarrow C\right)=\operatorname{deg}\left(D_{i j} \rightarrow C_{1}\right) \cdot \operatorname{deg}\left(D_{1} \rightarrow C\right) .
$$


Moreover, recalling that $n(X)=\operatorname{deg}\left(D_{1} \rightarrow C\right)=\operatorname{deg} \beta_{1}$, we get

$$
n(X)=\operatorname{deg}\left(\beta_{1}^{-1}\left(D_{i}\right) \rightarrow D_{i}\right)=\sum_{j} \operatorname{deg}\left(D_{i j} \rightarrow D_{i}\right)
$$

where $D_{i j}$ variies over all curves dominating $D_{i}$. Combining (3.2) and (3.3), we receive (3.1). Proceeding this way, we obtain a sequence $X_{n}$ of fibred etale covers. (3.1) shows that for $n>>0$ the surface $\tilde{X}=X_{n}$ and the fibration $\tilde{\pi}=\pi_{n}$ satisfy the requirement.

\section{The case ' $\operatorname{kod}(X)=-\infty, q(X)>0$ '}

In this section we are going to prove that no irregular surface in $\mathcal{T}$ has negative Kodaira dimension. We proceed by way of contradiction. Thus, assume that $X \in \mathcal{T}$ has negative Kodaira dimension. Then there exists a birational morphism $\phi: X \longrightarrow Y$ onto a relatively minimal model such that $Y$ is geometrically ruled over a curve $C$. Here, the genus of $C$ is the irregularity of $Y$ (cf. Subsection 2.1). So, $C$ is elliptic, because $C$ is dominated by components of $D$. We define $S_{i}, S$ to be $\phi\left(D_{i}\right), \phi(D)$ respectively. The next is clear from Section 3 .

Claim 4.1. It is sufficient to derive a contradiction in the case where the curves $S_{i}$ are all sections of the ruling.

We assume the hypotheses of Claim 4.1. We define $h_{X}$ to be the number of components of $D$. Note that by Lemma $3.2, h_{X}=S F \geq 3$ where $F$ is a general fibre of $\pi$. Next, we derive from Subsction 2.2:

Claim 4.2. We can assume that $D_{h_{X}}$ is not affected during the transition from $X$ to $Y$, i.e. that $S_{h_{X}}^{2}=D_{h_{X}}^{2}<0$ and $S_{i} S_{h_{X}}=0$ for all $i<h_{X}$.

With respect to numerical equivalence it holds then

$$
S_{i} \equiv S_{h_{X}}-S_{h_{X}}^{2} F\left(i<h_{X}\right)
$$

In fact, by Subsection $2.1 N u m(Y)=\mathbb{Z} S_{h_{X}} \oplus \mathbb{Z} F$, and we have $S_{i} \equiv S_{h_{X}}+b_{i} F$ with a $b_{i} \in \mathbb{Z}$. Hence, $b_{i}=S_{i} S_{h_{X}}-S_{h_{X}}^{2}=-S_{h_{X}}^{2}$. Note that this is up to now the second place where we use that the $S_{i}$ 's are sections. In particular, $S_{i} F=1$ and

$$
S_{i}^{2}=S_{h_{X}} S_{i}-S_{h_{X}}^{2}=-S_{h_{X}}^{2}>0\left(i<h_{X}\right)
$$

We also need to use the fact that $K_{Y}^{2}=c_{2}(Y)=0$ (cf. [1, p. 244]). Together with Tian-Yau's theorem 3.1 we find

$$
-D^{2}=4 s,
$$

where $s$ is the number of blow-ups in the transition from $Y$ to $X$. Recall that $K_{Y} S_{i}+$ $S_{i}^{2}=0$ for all $i$. Now, let $x$ vary over all points that are centers of blowing ups in the transition from $Y$ to $X$, and write $s_{x}$ for the multiplicity of the proper transform of $S$ at $x$. We apply [3, Ch. V, Exmpl. 3.9.2], the adjunction formula and Claim 4.2, and receive

$$
0=K_{X} D+D^{2}=K_{Y} S+S^{2}-\sum_{x} s_{x}\left(s_{x}-1\right)=\sum_{1 \leq i \neq j<h_{X}} S_{i} S_{j}-\sum_{x} s_{x}\left(s_{x}-1\right) .
$$


Combining with (4.1) and (4.2), and letting $t=-D_{h_{X}}^{2}=-S_{h_{X}}^{2}$, we deduce

$$
\left(h_{X}-1\right)\left(h_{X}-2\right) t=\sum_{x} s_{x}^{2}-\sum_{x} s_{x} .
$$

On the other hand, $\sum_{i<h_{X}} S_{i}^{2}=\left(h_{X}-1\right) t$ and for each $i$ the multiplicity $s_{i, x}$ of the proper transform of $S_{i}$ at $x$ equals 0 or 1 . By [3, Ch. V, Prop. 3.6], $D_{i}^{2}=S_{i}^{2}-\sum_{x} s_{i, x}^{2}$. With this and because of (4.3), we get

$$
4 s=-D_{h_{X}}^{2}-\sum_{i<h_{X}} D_{i}^{2}=t+\left(\sum_{i, x} s_{i, x}^{2}-\left(h_{X}-1\right) t\right)=-\left(h_{X}-2\right) t+\sum_{x} s_{x},
$$

i.e.

$$
\left(h_{X}-2\right) t=\sum_{x}\left(s_{x}-4\right) .
$$

Now, define $m_{3}$ to be the number of points $x$ such that $s_{x}=3$, and let $m_{4}$ be the number of points $x$ with $s_{x}=4$. Note that always $s_{x} \geq 3$, as results from Lemma 3.3 and the fact that the $\phi$-exceptional divisor consists of rational curves. So,

$$
\left(h_{X}-2\right) t=\sum_{x, s_{x} \geq 5} s_{x}-m_{3},
$$

that is,

$$
4 m_{3}+4 m_{4}+\left(h_{X}-2\right) t=\sum_{x} s_{x} \stackrel{(4.5)}{=} 4 s+\left(h_{X}-2\right) t .
$$

We conclude that $m_{3}+m_{4}=s$, so $3 \leq s_{x} \leq 4$ for all $x$. For the final step, we combine (4.4) with (4.5), and obtain

$$
\sum_{x}\left(s_{x}^{2}+4\left(h_{X}-1\right)-h_{X} s_{x}\right)=0 .
$$

As $3 \leq s_{x} \leq 4$, this yields

$$
m_{3}\left(5+h_{X}\right)+12 m_{4}=0,
$$

an obvious contradiction. We are done with this case.

\section{The Case ${ }^{\prime} \operatorname{kod}(X)=0, \mathbf{q}(X)>0 '$}

We start by proving:

Lemma 5.1. Let $Y$ be a complex irregular minimal surface with $\operatorname{kod}(Y)=0$. Then any two distinct elliptic curves $S_{1}, S_{2} \subset Y$ intersect each other transversally (if at all).

Proof. We know from Subsection 2.1 that $Y$ is either abelian or it is a hyperelliptic surface. If $Y$ is hyperelliptic then it admits an abelian etale covering, and we are therefore reduced to the case where $Y$ is abelian. Let $x \in S_{1} \cap S_{2}$. We may assume that $x$ serves as the neutral element of the underlying abstract groups on $S_{1}, S_{2}$ and $Y$. If $\iota_{i}: S_{i} \longrightarrow Y$ denotes the respective embedding for $i=1,2$ then the Lie functor yields a linear mapping

$$
\operatorname{Lie}_{\iota_{i}}: \operatorname{Lie}\left(S_{i}\right)=\mathbb{C} \longrightarrow \operatorname{Lie}(Y)=\mathbb{C}^{2} .
$$


A priori, the lines $\operatorname{Lie}_{\iota_{1}}(\mathbb{C})$ and $\operatorname{Lie}_{\iota_{2}}(\mathbb{C})$ are equal or intersect transversally in $0 \in \mathbb{C}^{2}$. By assumption, the second possiblity holds true. The result is now immediate.

With this in mind, let $Y$ be a minimal model of an irregular surface in $X \in \mathcal{T}$ and assume that $\operatorname{kod}(Y)=0$. By Subsection 2.1, $Y$ is either abelian or it is a hyperelliptic surface. By way of contradiction, assume the second mentioned possibility: $Y$ is hyperelliptic. Note that $X \neq Y$. Namely, $K_{Y}$ is numerically trivial and therefore $Y$ does not contain elliptic curves with negative self-intersection, whereas $X$ does (Cor. 3.2). Denote by $S_{i}$ the image curve of $D_{i}$.

Claim 5.2. Each curve $S_{i}$ is smooth elliptic.

Proof. Let $D=\sum D_{i}$ be the compactification divisor of $X$ and consider a birational morphism $\phi: X \longrightarrow Y$. Y admits an etale covering $\alpha: \tilde{Y} \longrightarrow Y$ by an abelian surface $\tilde{Y}$, and $\alpha$ induces an etale covering $\beta: \tilde{X} \longrightarrow X$. Let $\tilde{D}=\beta^{-1}(D)$. Then, $(\tilde{X}, \tilde{D})$ is a $\mathcal{T}$-pair, because $\tilde{X} \backslash \tilde{D}$ must have $\mathbf{B}$ as universal holomorphic covering. Let $\tilde{S}$ be the respective divisor on $\tilde{Y}$, i.e. $\tilde{S}=\alpha^{-1}(S)$. We know from Thm. 1.2 that $\tilde{S}$ is a sum of elliptic curves $\sum \tilde{S}_{i j}$ satisfying $4 \tilde{s}=\sum \tilde{s}_{i j}$ with $\tilde{s}=|\operatorname{sing} \tilde{S}|$ and $\tilde{s}_{i j}=\left|\tilde{S}_{i j} \cap \operatorname{sing} \tilde{S}\right|$ (the first index means that $\tilde{S}_{i j}$ dominates $S_{i}$ ). Let $U$ be a small ball around a point $x \in \operatorname{sing} \tilde{S}$. It results from Lemma 5.1 that the sheets $S \cap U$ become disjoint after blowing up once along $x$. Thus, it follows from Lemma 3.2 that $\tilde{X}$ results from $\tilde{Y}$ by blowing-up once at each point $x \in \operatorname{sing} \tilde{S}$; in particular, $\tilde{D}$ always intersects transversally with an exceptional curve. Therefore,

$$
K_{\tilde{X}}^{2}=K_{\tilde{Y}}^{2}-\tilde{s}=-\tilde{s}
$$

and

$$
c_{2}(\tilde{X})=c_{2}(\tilde{Y})+\tilde{s}=\tilde{s},
$$

so that $-\tilde{D}^{2}=4 \tilde{s}$ by Thm. 3.1. Moreover, since $\beta$ is etale, the single blowing ups along $M=\operatorname{sing} D$ produce $X$, and $\tilde{D}$ always intersects transversally with an exceptional curve.

Now, suppose that for some $i$ a point $x \in S_{i}$ is a singularity of $S$ with the property that for a small open ball $U \subset X$ around $x$ a sheet of $S_{i} \cap U$ admits a cusp singularity at $x$. Then the exceptional curve $L \subset X$ arising from the blow-up at $x$ does not intersect $D_{i}$ transversally. As $\beta$ is etale, neither do a $\tilde{D}_{i j}$ and an exceptional curve $\tilde{L} \subset \alpha^{-1}(L)$. By contraposition, no sheet of $S_{i} \cap U$ has cusp singularities. Therefore, if $\nu_{i}: D_{i} \longrightarrow S_{i}$ is the restriction of $\phi$ to $D_{i}$, then

$$
D_{i}^{2}=S_{i}^{2}-\left|\nu_{i}^{-1}\left(S_{i} \cap M\right)\right| .
$$

It still remains to show that each curve $S_{i}$ is indeed smooth. But, clearly, for $n=\operatorname{deg} \alpha$ we have $n \cdot \sum_{i}\left|\nu_{i}^{-1}\left(S_{i} \cap M\right)\right|=\sum_{i j} \tilde{s}_{i j}$. Since $n D^{2}=\tilde{D}^{2}=4 \tilde{s}$, we deduce

$$
-4 \cdot \frac{\tilde{s}}{n}=D^{2}=\sum_{i}\left(S_{i}^{2}-\left|\nu_{i}^{-1}\left(S_{i} \cap M\right)\right|\right)=\sum_{i} S_{i}^{2}-\frac{\sum_{i j} \tilde{s}_{i j}}{n} .
$$

Recalling that $4 \tilde{s}=\sum \tilde{s}_{i j}$ and noting that a priori $\left(K_{Y}+S_{i}\right) S_{i}=S_{i}^{2} \geq 0$, we conclude $\left(K_{Y}+S_{i}\right) S_{i}=0$. So, the claim follows from adjunction formula: $p_{a}\left(S_{i}\right)=1$. 
As already noticed above, $X$ results from $A$ by single blowing-ups along the points in $\operatorname{sing} S$. Since each component of $S$ is smooth by Lemma 5.1, it follows from Lemma 3.3 that there exists a point $y \in Y$ which is the intersection point of three distinct elliptic curves $S_{1}, S_{2}$, and $S_{3}$. On the other hand, the rank of $N u m(Y)$ is $\leq b_{2}(Y)=2$. Thus, for some numbers $a, b \in \mathbb{Q}$, we receive a numerical equivalence

$$
S_{1} \equiv a S_{2}+b S_{3} .
$$

Using that $S_{1}^{2}=S_{2}^{2}=S_{3}^{3}=0$, one readily derives a contradiction.

\section{References}

[1] Barth, W., Hulek, K., Peters, C, van de Ven, A., Compact Compl. Surfaces, second ed. (Springer, Berlin, 2004)

[2] Deligne, P., Mostow, G. D., Commensurabilities Among Lattices in PU(1,n), Annals of Mathematics Studies 132 (Princeton University Press, Princeton, NJ, 1993)

[3] Hartshorne, R., Algebraic geometry, GTM no. 52 (Springer, New York-Heidelberg, 1977)

[4] Holzapfel, R.-P., Ball and Surface Arithmetics, Aspects vol. E29 (Vieweg, Braunschweig, 1998)

[5] Holzapfel, R.-P., Abelian approach to modular forms of neat 2-ball lattices: Dimension formulas, HU-preprint (2001)

[6] Holzapfel, R.-P., Jacobi theta embedding of a hyperbolic 4-space with cusps, HU-Preprint (2001)

[7] Holzapfel, R.-P., Complex hyperbolic surfaces of abelian type, Serdica Math. J. 30 (2004), 207238

[8] Holzapfel, R.-P., Uludag, A. M., Yoshida, M. (ed.), Arithmetic and Geometry Around Hypergeometric Functions, Progress in Mathematics vol. 260 (Birkhäuser, Basel, 2007)

[9] Picard, E., Sur des fonctions de deux variables indépendantes analogoues aux fonctions modulaires, Acta math. 2 (1883), 114-135

[10] Tian, G., Yau, S.T., Existence of Kähler-Einstein metrics on complete Kähler manifolds and their applications to algebraic geometry, in: Yau (ed.), Mathematical Aspects of String Theory, Advanced Series in Mathematical Physics vol.1 (World Scientific, Singapore, 1987)

Departament Mathematik, ETH Zürich, HG G17, Rämistrasse 101, 8092 Zürich, SwitzerLAND

E-mail address: aleksander.momot@math.ethz.ch 